\title{
Evaluación de una Renovación Metodológica para un Aprendizaje Significativo de la Física
}

\author{
Iván R. Sánchez \\ Departamento de Física, Facultad de Ciencias, Universidad del Bío-Bío, Collao 1202, Casilla 5C, \\ Concepción-Chile (e-mail: isanchez@ubiobio.cl)
}

Recibido May. 07, 2012; Aceptado May. 25, 2012; Versión final recibida May. 30, 2012

\begin{abstract}
Resumen
Se analizan las implicancias didácticas de una renovación metodológica para un aprendizaje significativo de la Física a través de la resolución de problemas integradores y contextualizados por investigación. Se discute también el impacto de dicha renovación en el rendimiento académico y pensamiento crítico como indicadores de aprendizaje significativo. El punto de partida es la presentación de un problema integrador de contenido para resolver a lo largo del semestre. El problema integrador se divide en una serie de problemas más específicos relacionados entre sí, a resolver en el semestre, que incluyen actividades de aprendizaje que van desde la exploración a la transferencia de contenidos. Los resultados evidencian un mejoramiento del rendimiento académico y del pensamiento crítico.
\end{abstract}

Palabras clave: renovación metodológica, aprendizaje significativo, resolución de problemas, actividades de aprendizaje, pensamiento crítico

\section{Evaluation of a Methodological Renewal for the Meaningful Learning of Physics}

\begin{abstract}
The educational implications of methodological renewal for meaningful learning of Physics through problem solving and contextualized integrators for research are analyzed. Also, the impact of such methodological renewal on academic achievement and critical thinking as indicators of meaningful learning is discussed. The starting point is the presentation of a conceptintegrating problem to be solved during the semester. The concept-integrating problem is divided into a number of more specific related problems, including learning activities such as exploration to transfer content.The results show improvements on academic performance and on critical thinking.
\end{abstract}

Keywords: renewal of methodological, meaningful learning, problem solving, learning activities, critical thinking 


\section{INTRODUCCION}

Actualmente las Universidades a nivel internacional, y en particular las chilenas están insertas en transformaciones del sistema educativo y que el eje que da estructura al proceso educativo está centrado en la promoción del aprendizaje significativo del estudiante; sus experiencias de aprendizaje y su transformación, son el verdadero desafío de la educación superior, más imperiosa y urgente en el contexto de esta nueva generación de estudiantes. Según Sánchez et al., (2008, 2009 y 2011), este proceso de mejora debe ser interactivo y se debe sustentar en los siguientes principios: a) mayor implicación y autonomía del estudiante; b) utilización de metodologías activas de trabajo en equipo, tutorías, etc.; c) el docente debe ser un agente creador de escenarios u entornos de aprendizaje contextualizados que estimulen a los alumnos.

En este sentido, Sánchez (2009b), propone un modelo para la enseñanza de las habilidades del pensamiento que permite su transferencia a través de los dominios del conocimiento que considera cuatro componentes: el componente actitudinal o de la disposición; la instrucción y la práctica de las habilidades del pensamiento crítico; actividades de transferencia a través de los contextos y el componente meta/cognitivo usado para dirigir y evaluar el pensamiento, todas estas habilidades ayudan a enfrentar con éxito las situaciones problemáticas con autonomía y decisión. Como resultados surge el pensamiento crítico, como un pensamiento estratégico, que ayuda a desencadenar en los estudiantes competencias básicas para enfrentar diversas situaciones del ámbito académico, personal, cotidiano y los capacite para la construcción de su propio conocimiento (Sánchez, 2009b).

El pensamiento crítico es uno de los procesos cognitivos a desarrollar en la formación de un ingeniero, en la actualidad se encuentran una multitud de definiciones que intentan explicar estos procesos. Halpern $(1998,2003,2006)$, afirma, que las habilidades inherentes al pensamiento crítico pueden ser mejoradas por el proceso de enseñar y aprender en este contexto (Morales, 2011) muestra que es posible desarrollarlo a través del aprendizaje basado en problemas (ABP), de aquí surge la necesidad de establecer el impacto de una renovación metodológica en el pensamiento crítico y en el desarrollo de habilidades cognitivas tales como discriminar información, elaborar y valorar argumentos, juzgar correctamente eventos probabilísticos, analizar relaciones causales, disponer de buenas estrategias de toma de decisiones, poseer recursos para resolver ciertos problemas, para lo cual se debe crear entornos de aprendizaje para transferir estas habilidades a un contexto real del diario vivir, fuera de las situaciones de la sala de clases.

En la actualidad, los problemas de lápiz y papel son una actividad habitual en la clase de Física, cuyo valor formativo es ampliamente reconocido por los docentes de la especialidad; con esta actividad se promueve la adquisición del conocimiento y el aplicar los aspectos teóricos a situaciones prácticas específicas. Estas actividades ayudan al estudiante a aprender a aprender, permitiendo aplicar sus conocimientos en la resolución de problemas de la vida diaria (Pozo et al., 1994) y a promover el desarrollo del pensamiento creativo (Garrett, 1988, p. 225). La resolución de problemas es una de las características más relevantes de la enseñanza de la Física, su importancia en el contexto de aula también se ha trasladado al ámbito de la investigación del aprendizaje, encontrándonos con una de las líneas tradicionales más prolíficas y presentes. En España y Chile por ejemplo, se encontraron con la paradoja de que, frente a una enseñanza universitaria de la Física centrada en gran medida en la resolución de problemas, se deja el proceso de enseñar y aprender en manos de profesores que los resuelven mecánicamente en la pizarra (Perales y Salinas, 2004, Becerra, et al, 2005; Sánchez, 2009a).

Normalmente en las aulas no se enseña a resolver problemas, es decir, a enfrentarse a situaciones desconocidas, sino que los profesores explican soluciones perfectamente conocidas y que, por supuesto, no generan ningún tipo de dudas ni exigen tentativas. Al resolver estos problemas el profesor pretende que el estudiante vea con claridad el camino a seguir para resolverlo. Consecuentemente los estudiantes pueden aprender dicha solución y repetirla ante situaciones prácticamente idénticas, pero no aprenden a abordar un verdadero problema y cualquier cambio pequeño les supone dificultades insuperables, provocando manipulaciones no 
significativas de datos, fórmulas e incógnitas, que a menudo, lo llevan al abandono del problema y de la asignatura.

Lo anterior, trae como consecuencia generar enfoques educativos innovadores centrados en el estudiante y su aprendizaje, en los procesos de construcción de conocimientos y no sólo en su transmisión. En este marco de referencia se diseña y construye una renovación metodológica, centrada en el aprendizaje significativo a través de la resolución de un problema integrador y contextualizado (ASARPIC) por investigación, que se puede articular en una serie jerárquica de problemas más acotados en contenido cuya solución aporta a la solución del problema integrador (Herrera y Sánchez, 2009; Oñate y Sánchez, 2010; Sánchez et al, 2011).

En el ASARPIC se trabaja en el aula en grupos de 4 alumnos de forma colaborativa, que investigan los conceptos y contenidos desconocidos, para luego resolver las actividades de aprendizaje (A.A) abiertas y cerradas, programadas para trabajar cada contenido en profundidad y facilitar la adquisición de conocimientos. La finalidad es favorecer el aprendizaje significativo de conceptos, procedimientos y actitudes propias de la Física que se manifieste en una mejora del rendimiento académico y en el desarrollo del pensamiento crítico, en concordancia con las nuevas tendencias de la didáctica de las ciencias (Sánchez et al; 2008; Sánchez, et al, 2009).

El rol del docente en el ASARPIC es ser un mediador, que favorece la discusión (en la sesión de trabajo) en el grupo para negociar significado. El docente no es la autoridad del curso y los alumnos sólo se apoyarán en él para la búsqueda de información. Los problemas presentados son utilizados como base para identificar los contenidos necesarios para su estudio, de manera independiente o en grupo, teniendo presente que el objetivo no es resolver el problema, ya que estos son utilizados para gatillar la necesidad de cubrir los objetivos de aprendizaje de las unidades programáticas de la asignatura a enseñar (Ribeiro y Mizukami, 2005; Said et al., 2005; Sánchez et al., 2008). A lo largo del trabajo los alumnos en grupo, van: adquiriendo responsabilidad y confianza a través de las actividades de aprendizaje a resolver, desarrollando la habilidad de criticar y ser criticado, lo que lleva a una mejora de su desempeño en el grupo.

EI ASARPIC, presenta un paradigma alternativo al tradicional que se enmarca dentro de un modelo educativo centrado en el aprendizaje, que permite a los estudiantes: adquirir conceptos y aplicarlos a nuevas situaciones, conseguir información de diversas fuentes y recursos; jugar un rol activo en el proceso de buscar alternativas, investigar, proponer soluciones y analizar situaciones de manera colaborativa, sirviéndose de las ventajas que le ofrecen las nuevas tecnologías de la información y de la comunicación. Se postula que este modelo responde más a las necesidades del mundo del trabajo, al contexto social y a las propias características de los estudiantes.

EI ASARPIC, implica una evolución del sistema educativo actual hacia uno más activo y participativo, centrando en el aprendizaje, que se logra a través de escenarios creados por el profesor en base a problemas, donde los alumnos, el profesor y el material educativo (problemas y actividades de aprendizaje) interactúan constantemente, se cree que ésta forma de trabajo y experimentación merece sin duda un esfuerzo adicional de búsqueda de información e investigación.

El problema integrador y los problemas más específicos son diseñados a partir de una noticia o situación del mundo real, éste debe integrar el máximo contenido posible; a partir de él se reconocen los contenidos que se tratarán en el curso, y se diseñan nuevos problemas más específicos entramados dentro del gran problema (Sánchez, 2009a). Estos problemas más específicos son presentados en secuencia para lograr abarcar la mayor cantidad de contenidos del programa del curso. Abordar estas situaciones implica identificar las ideas previas y los contenidos que cada alumno o grupo de alumnos deben investigar. Además, se debe tener presente la resolución de actividades de aprendizaje para la apropiación de los contenidos, que permita resolver el problema integrador.

Es preciso subrayar que, en la propuesta, no se concibe a los alumnos y alumnas como investigadores autónomos trabajando en la frontera del conocimiento (ni tampoco como simples 
receptores), pues esto presenta graves limitaciones y no resulta útil para organizar el trabajo de los estudiantes. Una mejor comprensión y adquisición de conocimiento, por ende de aprendizaje en el aula, se logra al concebir a los estudiantes con un rol de investigadores, ordenados en equipos cooperativos, que abordan problemas de interés, interaccionando con los otros equipos y con el resto de la comunidad científica, representada por el profesor y los libros de textos.

Los resultados alcanzados después de 8 semestres de aplicar el ASARPIC para enseñar y aprender (E-A) los contenidos de Física I a los estudiantes de Ingeniería de la Universidad del Bío-Bío, Concepción-Chile, han sido positivos, los alumnos se muestran motivados y opinan favorablemente sobre esta forma de trabajo; por otra parte la investigación reporta cambios significativos en el rendimiento académico y pensamiento crítico, que se manifiestan con un mayor grado de satisfacción y compromiso con su aprendizaje, de modo que su implementación resulta recomendable en cualquier otro campo del saber.

\section{Aprendizaje Significativo y resolución de problema}

Durante los últimos años, múltiples investigaciones en el proceso de E-A manifiestan la importancia de las ideas previas de los alumnos (Driver et al., 1994; Ausubel, 2000; Moreira, 2005; Sánchez et al., 2005. Sánchez, et al., 2008; Sánchez et al., 2009; Sánchez et al., 2011). En estas propuestas de renovación metodológica, es preciso identificar y considerar las ideas con que los estudiantes ingresan al aula y los contenidos a enseñar, para seleccionar y organizar las AA que se van a diseñar, y elaborar para que favorezcan el desarrollar el aprendizaje significativo. La mayoría de las ideas alternativas tienen sentido para los estudiantes y son útiles para dar explicaciones, finalmente arraigadas en su estructura cognitiva y son resistentes al cambio.

La innovación de la propuesta pretende facilitar el construir aprendizaje significativo a través de resolver un problema integrador de contenido por investigación en lo posible contextualizado a la especialidad de los estudiantes, eje conductor de la asignatura; que lleva a identificar y estructurar los contenidos y los procedimientos de la disciplina, a través de la solución de una serie jerárquica de problemas más acotados, para avanzar en la solución del problema integrador. A partir de la implementación de la propuesta se pretende desarrollar un proceso educativo más activo y participativo centrado en el aprendizaje, donde el alumno asuma la responsabilidad de aprender y el profesor asuma el rol de crear entornos de aprendizaje colaborativos para la interacción, orientar y compartir significado con el alumno.

Para Moreira (2005) el aprendizaje significativo se caracteriza por una interacción entre las ideas relevantes existentes en la estructura cognitiva y las nuevas informaciones, a través de las cuales éstas adquieren significados y se integran en la estructura cognitiva de manera no arbitraria y sustancial, contribuyendo a la diferenciación, elaboración y estabilidad de los inclusores existentes. La estructura cognitiva existente juega en el ASARPIC, un papel decisivo. Lo que corrobora el hecho de que la solución de cualquier problema dado, supone la reorganización del residuo de las experiencias previas, de modo que se ajuste a los requisitos concretos de la tarea planteada. Si los conocimientos previos existentes en la estructura cognitiva (conceptos, principios, leyes, etc.) "son claros, estables y discriminables, facilitan la resolución de problemas. Sin tales conocimientos no es posible de hecho, ninguna resolución de problemas, independientemente del grado de destreza que el alumno tenga en materia de aprendizaje por descubrimiento; sin tal conocimiento, ni siquiera podría empezar a entender la naturaleza del problema que enfrenta" (Ausubel et al., 1997, p. 490; Ausubel, 2000; Moreira, 2010).

En Física, como en otras disciplinas, la simple memorización de ecuaciones, leyes y conceptos puede tomarse como ejemplo típico de aprendizaje superficial y reiterativo. Otro ejemplo es el de los alumnos que no consiguen resolver problemas o cuestiones que impliquen usar o transferir ese conocimiento y que argumentan que "lo saben todo", pero en el momento de la evaluación no responden (Sánchez y Flores, 2004).

Para Moreira $(2005,2006)$, otra condición necesaria para el aprendizaje significativo es que el alumno manifieste disposición para relacionar, de manera sustantiva y no arbitraria, el nuevo 
material, con su estructura cognitiva. Esta condición indica que, independientemente si el material a E-A es potencialmente significativo, si la intención del estudiante es memorizarlo, tanto el proceso de aprendizaje como su producto serán superficiales y reiterativos. Un material preparado para enseñar y aprender no es significativo en sí mismo, sólo es significativo cuando entra en interacción con las estructuras cognitivas de los estudiantes, pero puede ser potencialmente significativo si presenta buena diferenciación entre los conceptos, adecuada organización jerárquica y una estructura clara en sus relaciones lo que logra a través del ASARPIC, donde los problemas a resolver provienen de noticias o contextos conocidos de la especialidad y dan cuenta de conceptos o ideas previas relevantes en la estructura cognitiva del estudiante, condición necesaria para que el material presentado sea potencialmente significativo generando una mayor posibilidad de manifestar disposición para relacionar, de manera sustantiva y no arbitraria, el nuevo material (Moreira, 2010).

El principio de la reconciliación integradora establece que al programar material de enseñanza, con base en un problema integrador de contenido y una serie de problemas más acotados, favorece la adquisición de aprendizaje significativo y evita fragmentar o separar ideas en temas particulares dentro de respectivos capítulos o subcapítulos; este enfoque favorece el principio de diferenciación progresiva de los conceptos o contenidos del curso, que al abordarlos por medio de problemas más acotados se van diferenciando en el transcurso de la instrucción del semestre.

Por otra parte, las prácticas en ASARPIC se entienden como situaciones de interacción social donde se trabaja de forma colaborativa, el lenguaje es imprescindible para comunicar, compartir experiencias y objetivos, a la vez puede dirigir guiar, orientar, acompañar la acción, ayudando a explicar y a hacer consciente el proceso, así los sujetos más competentes ayudan a los menos competentes a utilizar de manera adecuada los sistemas de signos con propósitos determinados (Saenz et al., 1998, p.151). El enfoque socio-cultural, derivado de la teoría de Vygotsky (1979), aporta una determinada manera de ver y entender la enseñanza, el aprendizaje y el desarrollo de la persona como procesos que van unidos, configurando el proceso socializador de los sujetos.

Mediante la interacción y la actividad compartida, el docente procura la ayuda necesaria en el aula, para que el estudiante se apropie progresivamente del conocimiento. Así, muestra al estudiante modelos, le proporciona información adicional, reduciendo el grado de dificultad, para disminuir progresivamente la ayuda y asegurar el traspaso del control del proceso de aprendizaje del docente al estudiante. Partiendo de los postulados de Vygotsky (1979) cabe destacar el papel del docente en el proceso de aprender, ofreciendo una labor de andamiaje que apoyará al estudiante en su aprendizaje. Para entender el concepto de andamiaje, es preciso hacer referencia a otro punto clave en la teoría, al concepto que él denomina Zona de Desarrollo Próximo (ZDP). Que "no es otra cosa que la distancia entre el nivel real de desarrollo, determinado por la capacidad de resolver independientemente un problema, y el nivel de desarrollo potencial, determinado a través de la resolución de un problema bajo la guía de un experto o en colaboración con otro compañero más capaz" (Vygotsky, 1979, p.133).

En el ASARPIC, la responsabilidad del aprendizaje se "traspasa" y corresponde al estudiante, que es el encargado de construir significados, de aquí se desprende que el proceso de aprendizaje se concibe como una construcción personal, mediada por la interacción social y por los signos, luego, el E-A se concibe como un proceso comunicativo, una construcción conjunta que implica negociación de significados, donde el rol del docente es crear entornos de aprendizaje que promuevan la participación activa de los estudiantes, para así favorecer el control de su propio aprendizaje, creando instancias de interacciones múltiples, a través de la triada profesor, material educativo y alumno (Novak y Gowin, 1988); con el objetivo de compartir significado en la clase, fomentando la naturaleza social del aprendizaje (Vygotsky, 1979).

De acuerdo a resultados previos obtenidos, Sánchez, (2009a) y Sánchez et al. $(2009,2011)$, se afirma que el empleo de la metodología bajo problemas por investigación, produce un mejoramiento en las prácticas pedagógicas apuntando hacia el logro de aprendizaje más significativo. Por otra parte, desarrolla capacidades para el trabajo en equipo, de comunicación oral y escrita de los alumnos. Si bien ASARPIC puede ser implementado de muchas formas en el 
aula, es común utilizar una secuencia de tres etapas. a) se presenta al equipo un texto que describe una situación y ellos deben definir las preguntas de investigación, los objetivos de aprendizaje y asignar tareas a investigar para cada integrante del equipo; b) los alumnos de forma individual investigan las materias asignadas; y c) en el grupo se socializa los contenidos investigados. Se regresa a la primera etapa para una nueva iteración hasta obtener los elementos necesarios para resolver las AA y los problemas (Duch et al. 2004, Sánchez, 2009a).

Una de las principales características del ASARPIC, según Sánchez et al., (2009) es fomentar en el alumno la actitud positiva hacia el aprendizaje significativo. En ella se promueve la autonomía del alumno, quien aprende a través de los contenidos y la propia experiencia de trabajo en el aula (dinámica del método). Los alumnos tienen además la posibilidad de observar en la práctica, aplicaciones de los aprendizajes en torno al problema. La transferencia pasiva de información es algo que se elimina en el ASARPIC; toda la información que se vierte en el grupo es investigada, aportada, o bien, generada por el mismo grupo. A continuación se describen algunas características del ASARPIC: Es un método de trabajo activo donde los alumnos participan constantemente en la adquisición de su conocimiento. El método se orienta a la solución de problemas que son seleccionados o diseñados a partir de noticias, periódicos, etc. Es un método que promueve el trabajo de forma colaborativa en grupos pequeños; se aprende a través de los contenidos y la propia experiencia de trabajo en el aula (dinámica del método); la actividad gira en torno a la discusión de un problema; el aprendizaje se adquiere de la experiencia de trabajar sobre ese problema; el aprendizaje es asumido por el alumno y no por el profesor; fomenta en el alumno la actitud positiva hacia el aprendizaje y su autonomía; el docente juega un rol de guía o mediador, su función es negociar significado.

A partir de estas características, Sánchez et al., (2009) afirma que el método estimula el autoaprendizaje y la práctica del estudiante al enfrentarlo a situaciones nuevas y reales, identificando su deficiencia de conocimiento. La experiencia de trabajar en grupos pequeños, orientado a la solución del problema, es característica distintiva del ASARPIC. En estas actividades al trabajar en grupos, los alumnos toman responsabilidades y acciones que son básicas en su proceso formativo.

Con la presentación del problema integrador se inicia el estudio de la Mecánica, identificando los contenidos a investigar y abordar, lo que se realiza a través de una lluvia de ideas, de donde surgen nuevos problemas más específicos a resolver, en los cuales es necesario identificar los conocimientos previos y los contenidos a investigar a partir del problema integrador, que puede ser la generación de electricidad en una central hidroeléctrica, construcción de Carreteras, funcionamiento de una Industria; etc. y su funcionamiento en los alrededores de la ciudad de Concepción. Enseguida se debe crear los escenarios de aprendizaje por contenidos y que son específicos; a través de estos problemas más acotados se trabaja en secuencia jerárquica, para alcanzar la solución del problema abarcador (Sánchez et al., 2008; Sánchez et al., 2009; Sánchez, 2009). Para alcanzar la solución de cada problema propuesto y lograr el aprendizaje significativo de los contenidos, según Sánchez, et al. (2009). se debe seguir el siguiente programa de actividades: a) Planteamiento del Problema, b) Aclarar conceptos y términos que no se entienden, identificando conocimientos previos, c) Definir y analizar problema con puesta en común, d) Asignación del contenido a investigar, e) Solución cualitativa, e) Búsqueda de información en forma individual, f) Trabajo colaborativo, compartiendo significado de contenido investigado, g) comunicación de la información realizado síntesis de información.

La propuesta de renovación metodológica ASARPIC presenta ejes estructuradores que se muestran en la Figura 1, la resolución de problemas, como medios para facilitar la adquisición del aprendizaje significativo y la interacción Social, donde se incluyen las características del trabajo científico y trabajo colaborativo necesarias para compartir significado. También, se consideran actividades de aprendizaje que van desde la exploración a la transferencia de contenidos. 


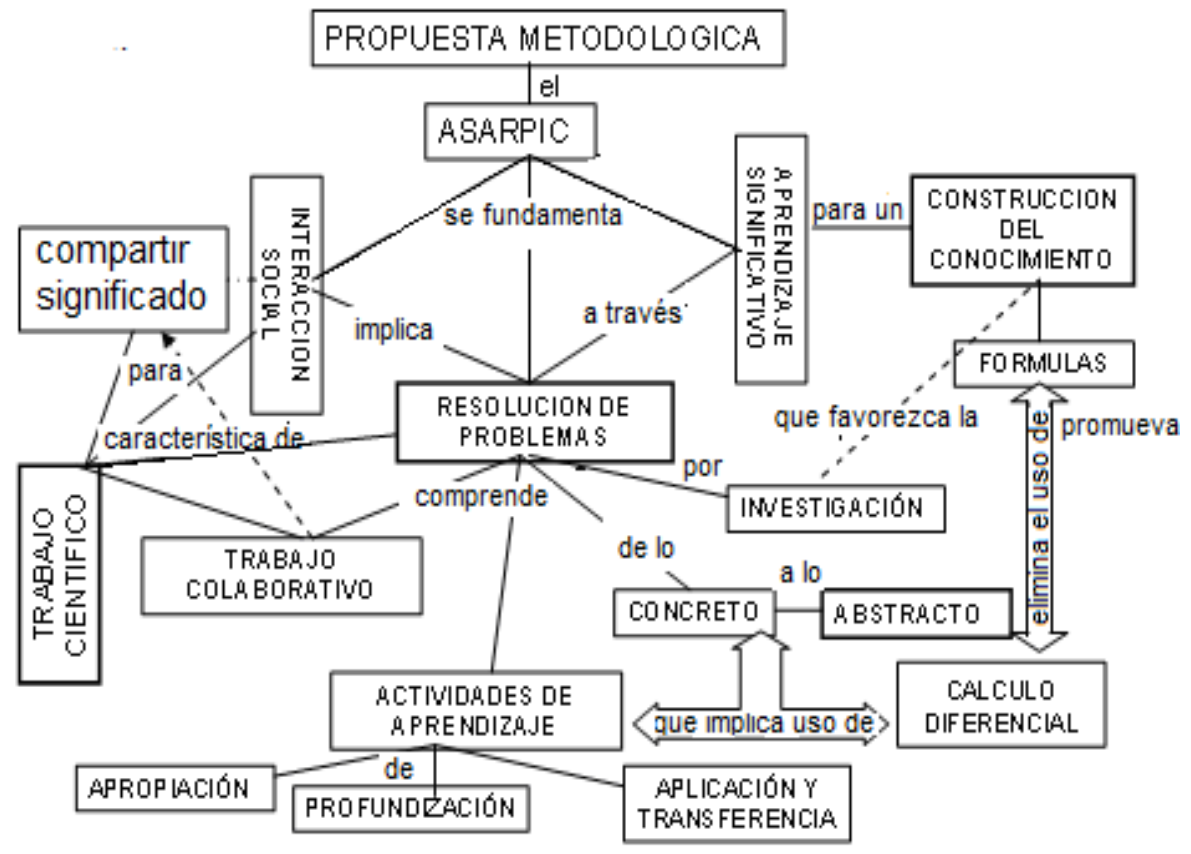

Fig. 1: Mapa conceptual que ilustra la propuesta de innovación metodológica de aula aplicada con base en problemas a resolver en el aula (Sánchez, 2007)

La dinámica del método en el aula para enseñar y aprender Física de forma activa y participativa (ASARPIC), se ilustra en la Figura 2, donde se promueve el trabajo colaborativo y la interacción social al resolver problemas que por una parte, son contextualizados de una noticia del mundo real (de un periódico, revista, TV. etc.) que en lo posible sea motivadora y facilite la interacción entre las ideas nuevas y los nuevos conocimientos condición necesaria para el aprendizaje significativo, y por otra parte, es el alumno el que resuelve los problemas investigando uno o mas contenidos en grupos y compartiendo significado a través del trabajo colaborativo, lo que promueve la interacción social, el compartir significado y desarrollar aprendizaje significativo.

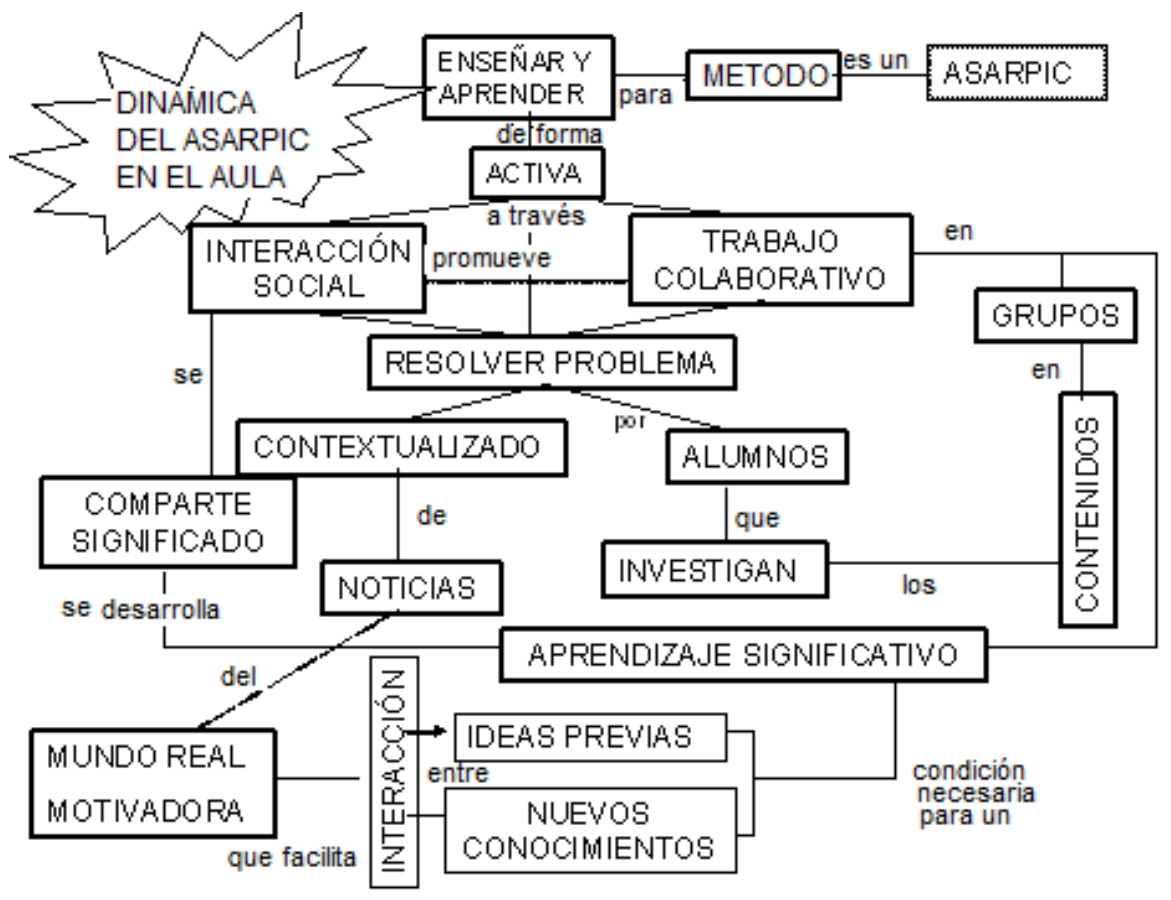

Fig. 2: Mapa conceptual de la dinámica de la propuesta con bases en problemas a resolver en el aula (Sánchez et al., 2009) 
Dentro de la experiencia de trabajar ASARPIC, se observa que los estudiantes van integrando estrategias de procesamiento de la información propias para la adquisición de conocimiento y aprenden sobre su propio proceso de aprendizaje. De aquí, se puede aseverar que los conocimientos en esta propuesta son introducidos en directa relación con el problema y no de manera aislada o fragmentada. Ésta se basa en la utilización de problemas, adecuadamente formulados, para motivar a los estudiantes a identificar, investigar y aprender los conceptos y principios que ellos necesitan conocer para alcanzar la solución, lo que lleva a desarrollar las competencias laborales, a trabajar en equipos de investigación, adquiriendo habilidades de comunicación e integración de información, luego se puede inferir que la propuesta genera: a) pensamiento crítico, capacidad para analizar y resolver problemas del mundo real, b) capacidades para encontrar, evaluar y usar apropiadamente los recursos de aprendizaje, c) trabajar colaborativamente en equipos, d) demostrar versatilidad y habilidades de comunicación efectiva, en forma oral y escrita, e) usar el conocimiento adquirido para aprendizaje continuo.

Dadas las razones necesarias para la renovación metodológica y sus efectos en el proceso de EA, conceptos, procedimientos, leyes y principios en Física I, se realizó una investigación comparativa entre dos metodologías: a) Tradicional, expositiva de transmisión acabada de conocimiento, y b) ASARPIC que considera la resolución de problemas por investigación y trabajo colaborativo. En la investigación se pretende establecer la influencia de la metodología en el rendimiento académico, en cada uno de las categorías del pensamiento crítico.

\section{METODOLOGÍA Y DISEÑO DE LA INVESTIGACIÓN}

Dos grupos de alumnos de Ingeniería, que cursan la asignatura de Física General I de la Universidad del Bío-Bío, reciben una intervención pedagógica de aula distinta en los mismos contenidos simultáneamente y con igual secuencia, el grupo control (GC) aborda los contenidos de forma tradicional, clase expositiva de transmisión acabada de conocimientos y en el grupo experimental (GE) las clases se realizan en base a la resolución de un problema integrador de contenido (ASARPIC) y una secuencia jerárquica de problemas más acotados, ambos grupos son independientes. Los resultados obtenidos a través de instrumentos validados que entregan datos que se pueden considerar como medidas de intervalos y se comparan bajo un diseño cuasiexperimental (Sierra, 2002) de investigación, que cumple con los supuestos exigidos por el diseño, la homogeneidad de varianza y normalidad. La investigación se realiza en el horario habitual de clases, durante las horas pedagógicas asignadas por su cuadro curricular por semana, que son los que corresponden a la asignatura.

\section{Instrumentos de recogida de la información}

1) Rendimiento académico: se mide por medio del rendimiento académico del semestre, a través de evaluación de carácter formal (dos Tests, dos Certámenes) elaborados por un conjunto o equipo de 3 a 4 docentes que trabajan la asignatura con metodología tradicional y experimental, lo que asegura la validez del contenido. La confiabilidad de cada uno de los instrumentos de medida se determina a través de la prueba de Kuder Richardson (KR20) o el coeficiente alpha de Cronbach.

2) Test de pensamiento crítico: El test, utiliza situaciones cotidianas y similares a las encontradas en la vida real con un doble formato de pregunta. Así, se plantea una situación problemática sobre la cual se formula una pregunta abierta y se pide que se seleccione la alternativa que mejor resuelve la cuestión planteada. El test consiste de 25 preguntas, cada una de las cuales requiere la construcción de respuestas abiertas, en una primera parte, luego por preguntas específicas, de respuestas de elección forzada que evalúan el razonamiento subyacente a la primera respuesta del estudiante. Las respuestas abiertas del estudiante reflejan el uso espontáneo de las habilidades de pensamiento crítico y las respuestas de elección forzada, muestran el grado en que se reconoce el problema cuando se le proporcionan algunas pistas. El test en su versión española esta validado (Nieto et al., 2009) y sus dimensiones según Halpern, $(2003,2006)$ son: a) Habilidades de razonamiento verbal $(\mathrm{V})$, b) Habilidades de análisis de argumento (A), c) Habilidades de pensar, probando hipótesis $(H), d)$ Uso de la probabilidad y la incertidumbre $(P), e)$ Habilidades de resolución de problemas y toma de decisiones (RP). 


\section{Muestra}

La muestra que se utiliza para poner a prueba la hipótesis, la constituyen 104 estudiantes de los cuales el $23 \%$ fueron mujeres y el $77 \%$ varones, cuya edad promedio fue de 20 años de la carrera Ingeniería civil que cursan Física General I de la Universidad del Bío-Bío de Concepción. El muestreo es del tipo aleatorio simple, en el que todos los alumnos tienen la misma probabilidad de ser seleccionadas como grupo experimental o control. Los estudiantes se inscriben vía Internet, hasta completar los cupos de cada curso y se abre una nueva sección, que será trabajada por otro docente, cualquiera de los dos grupos puede ser experimental o control.

\section{Análisis de los datos}

Para establecer el nivel de significado debido al tratamiento estadístico de los datos, se usa; la prueba de Kuder Richardson KR20 o alpha de Cronbach, para la fiabilidad y confiabilidad de los instrumentos de medidas (López et al., 1997 y Sierra, 2002), para determinar si una o unas variables se comportan aproximadamente de forma normal, se utiliza la prueba de KolmogorovSmirnov y para el estudio de la homogeneidad de varianzas poblacionales se utiliza la de Prueba de Levene. Para establecer si existen diferencias estadísticamente significativa entre los grupos y entre un mismo grupo se emplea la prueba $t$ de Student. Además se realiza un estudio descriptivo para describir la relación entre variables, por medio del Análisis de Correspondencia Múltiple (ACM) que representa gráficamente las relaciones de dependencia existentes entre las diversas modalidades de las variables categóricas en estudio. Para el análisis del rendimiento académico y categorías del pensamiento crítico, se emplea además, la prueba de la media, y el método univariado (Cohen y Manion, 1990), de representaciones gráficas a través del porcentaje.

\section{RESULTADOS}

\section{a) Test de Pensamiento Crítico de Halpern en dos mediciones.}

Al estudiar la normalidad de los datos obtenidos como resultados de los test de los GE y GC, se puede concluir que los puntajes del pre-test y del pos-test tienen un nivel de significación asintótica grande, por lo tanto, no es posible rechazar la normalidad de los datos. Se concluye que los puntajes obtenidos en el test de pensamiento crítico Halpern se ajustan asintóticamente a una distribución normal de parámetros según la Prueba de Kolmogorow - Smirnow: GC y GE que entregan una Media y Desviación en el: pre-test $(\mu=57,60 ; \sigma=30,47)$ y en el pos-test ( $\mu=81,99, \quad \sigma=34,86)$ respectivamente.

Para establecer la Homogeneidad de varianza de los datos se usa la prueba estadística Levene, que entrega un estadístico $\mathrm{F}$ para el pre-test y pos-test; de valor crítico 1,8 para un nivel de significancia $p=0,05$. Del análisis de los puntajes del pre-test del GC y GE se obtiene un $F=1,731$ que es menor al valor crítico $(F=1,8)$ de aquí se establece que varianza son iguales en la primera medición, no existiendo diferencias estadísticamente significativas entre los grupos; al estudiar los puntajes obtenidos en el pos-test se obtiene un valor de $\mathrm{F}=4,598$ que es mayor al valor crítico obteniéndose un nivel de significado menor al 0,05 , de donde se infiere que las varianzas son distintas, existiendo diferencias estadísticamente significativas a favor del GE.

Al analizar los resultados obtenidos en el Test Halpern se observa que el puntaje más bajo corresponde al pre-test de un estudiante del GC y uno del GE que obtuvieron cero puntos, por el contrario el puntaje más alto corresponde a dos estudiantes del GE, quien obtuvo 144 puntos en el post-test. Esto muestra una diferencia de 50 puntos respecto del máximo ideal de este test que corresponde a 194 puntos. La figura 3, muestra los promedios del pre-test y post-test para ambos grupos, de aquí se puede inferir preliminarmente que los resultados de ambos test en el GE son superiores a los del GC. También se advierte que la diferencia promedio entre pre-test y post-test es mayor en el GE que en el GC. Lo que permitirá afirmar que la renovación metodológica ASARPIC usada en el grupo experimental tiene un mayor impacto. Esto se corrobora por medio de la prueba estadística t de Student para el GE y GC que obtienen un estadístico $t=12,95$ y un nivel de significancia $p=0,0000(99,99 . . \%)$ para el GE y un estadístico $t=9,422$ con un nivel de significancia $p=0.000(99,9 . \%)$ para el GC, lo que muestra cambios estadísticamente significativo entre los resultados obtenidos entre el pre y post test de cada grupo. 


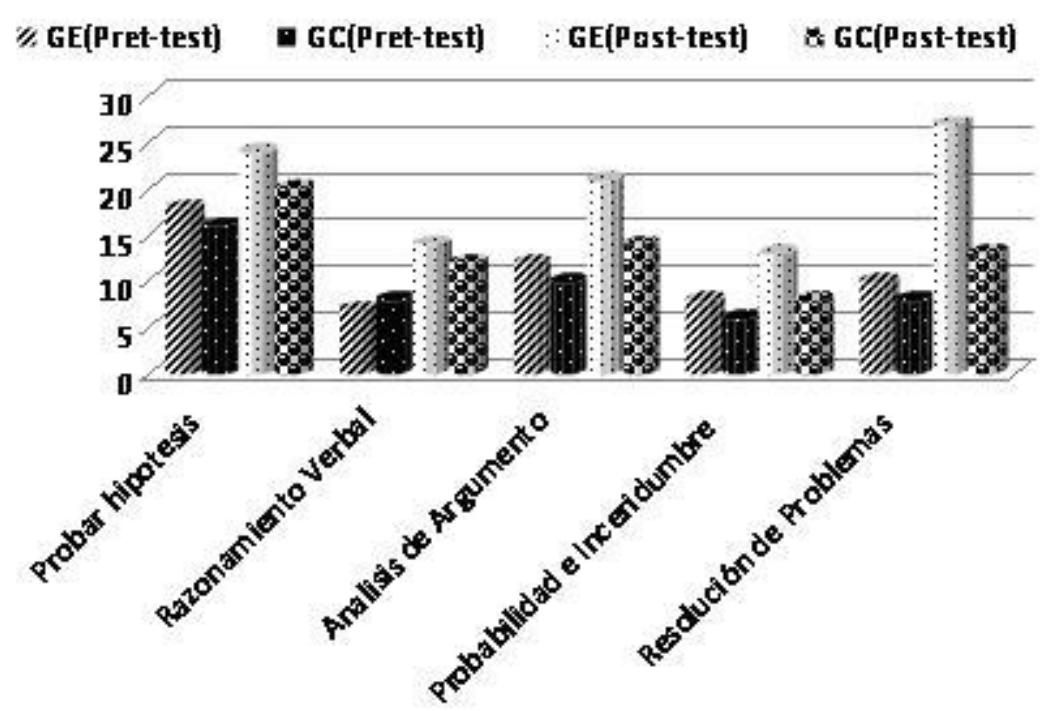

Fig. 3: Resultados por categoría test de Halpern en dos mediciones

De la figura 3; se observa que el GE en el pre - test obtiene una media más alta en las habilidades de: probar hipótesis, análisis de argumento, probabilidad e incertidumbre y resolución de problemas en cambio el GC obtiene una media más alta en la habilidad de razonamiento verbal en el pre-test. En cambio en el post-test segunda medición el GE obtiene una media o promedio mayor en las cinco habilidades que forman la estructura del test que el GC. Del análisis de ambos grupos en el post-test, se puede ver que sólo en una de las cinco habilidades que conforman la estructura del test pensamiento crítico de Halpern, habilidad de razonamiento verbal, los puntajes del GC pre-test son superiores a los del GE, en el post-test los resultados del GE son similares o superiores al GC. Del análisis estadístico de comparación de media para dos muestra independiente que se realiza por la t de Student se obtiene un estadístico $t=10,48$ con un nivel de significancia de $p=0,000(99,995 \%)$, de donde se infiere que existen diferencias estadísticamente significativas a favor del grupo experimental al $99.995 \%$.

\section{b) Rendimiento académico}

En la figura 4, se representa los resultados obtenidos del análisis de los rendimientos académicos del GC y GE al final del semestre después de ser enfrentados a diversas evaluaciones test y certámenes. Se debe tener presente que antes de la intervención no se encuentran diferencias estadísticamente significativas, entre los GC y GE, test conocimientos previos; en cambio al revisar los rendimientos después de las respectivas intervenciones al final del semestre, se encuentran diferencias en el rendimiento académico a favor del GE.

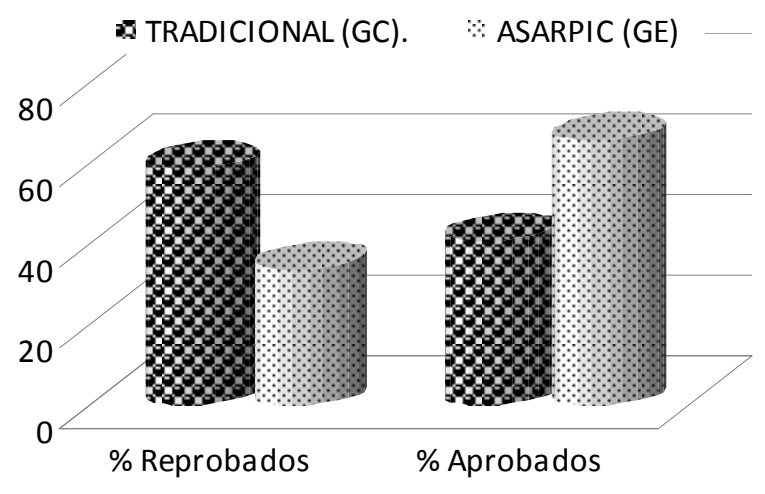

Fig. 4: Muestra rendimiento académico final GC y GE con diferencias significativas $p=0,028$. 
Al interpretar la figura 4, se observa que el mayor número de estudiantes aprobados es del GE y el mayor número de alumnos reprobados es del GC, lo anterior se corrobora por medio de la prueba $t$ de Student que entrega con un estadístico $t=9,23$, y un nivel de significados $p=0,000$ $(99,9, \%)$ que establece diferencias estadísticamente significativas a favor del grupo experimental de después de la intervención metodológica.

\section{c) Análisis de correspondencia múltiple (ACM) cuestionario pensamiento crítico}

El análisis de correspondencias múltiple (ACM), para el grupo que trabaja con ASARPIC, se realizó aplicando un paquete estadístico "STATISTICA 6.0" para Windows, con la finalidad de representar gráficamente las relaciones de dependencia existentes entre las diversas modalidades de las variables (dos o más) categóricas en estudio, a partir de la información proporcionada por tablas de frecuencias cruzadas En este caso, las variables en estudio e involucradas en la investigación son las siguientes: habilidades de razonamiento verbal $(V)$, habilidades de análisis de argumento $(A)$, habilidades para pensar probando hipótesis $(H)$, uso de la probabilidad y la incertidumbre $(P)$, habilidades para la toma de decisiones y resolución de problemas (RP) y rendimiento académico (NF) con $A$ : aprobado o R: reprobado. Estas variables se clasifican en dos categorías: sobre la normal (SN) y bajo la normal (BN).

En el presente se han considerado los tres primeros ejes factoriales, para explicar la dependencia entre las variables ya que éstos acumulan el $92 \%$ de la variabilidad total de la nube. En la figura 5, se muestra las primera dos dimensiones.

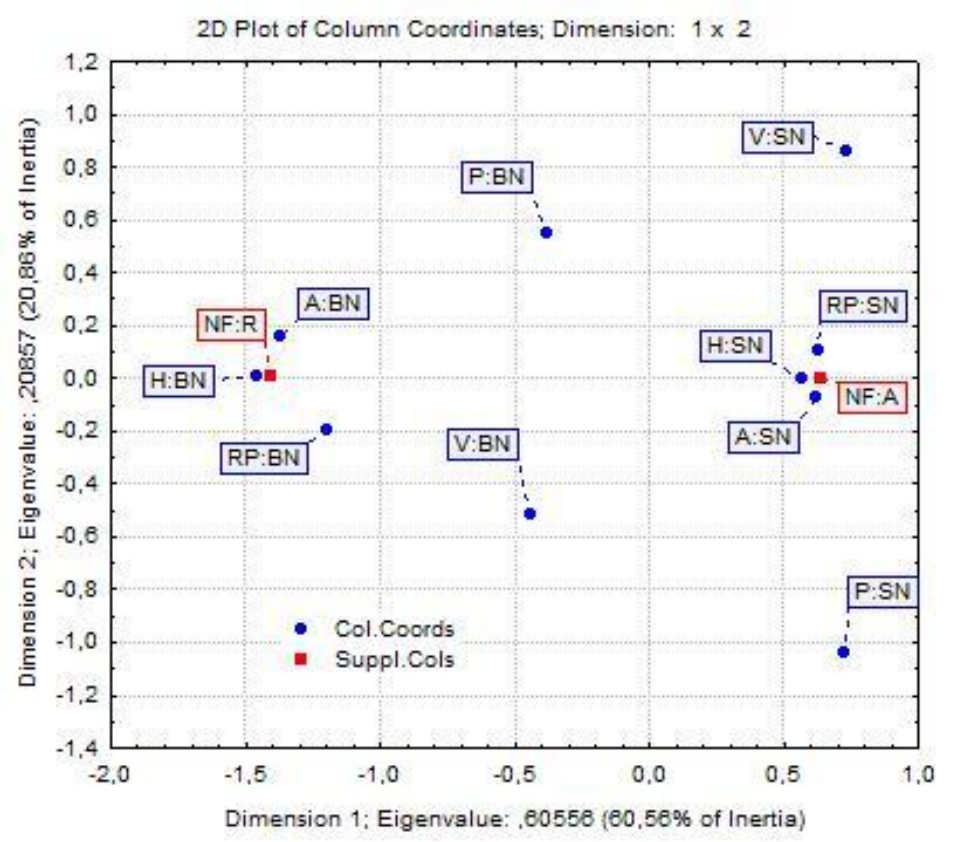

Fig. 5: Muestra la relación entre las variables que afectan el rendimiento académico

De la figura 5 , se desprende que en la dimensión 1 , se caracteriza por los alumnos aprobados NFA son las categorías del test de pensamiento crítico que sobre la normal ASN; HSN, RPSN y en menor medidas VSN, a estas características se le contrapone un bajo rendimiento académico que se caracteriza por tener ABN, HBN, RPBN y en menor medida VBN y PBN. La dimensión dos entrega muy poca información y las variables que más aporta en este eje son VSN a las que se contraponen VBN que están alejadas del rendimiento, en menor medida entrega información de la variable PSN a la que se contrapone PBN. De aquí se desprende que el pensamiento crítico es dirigido, razonado y propositivo que se centra en la comprensión de la situación en cuestión, la formulación de hipótesis, la toma de decisiones, la resolución de problemas, el cálculo de probabilidad y la evaluación de su propio proceso. 
Al estudiar la relación entre la primera y tercera dimensión se observa que las variables que más contribuyen a la formación de esta dimensión o eje factorial son la actividad NFA, RPSN y VSN. Ambos ejes recogen casi el $71 \%$ de la variabilidad de los factores. La información recogida en las dimensiones 1 y 2 se refleja, en este tercer factor.

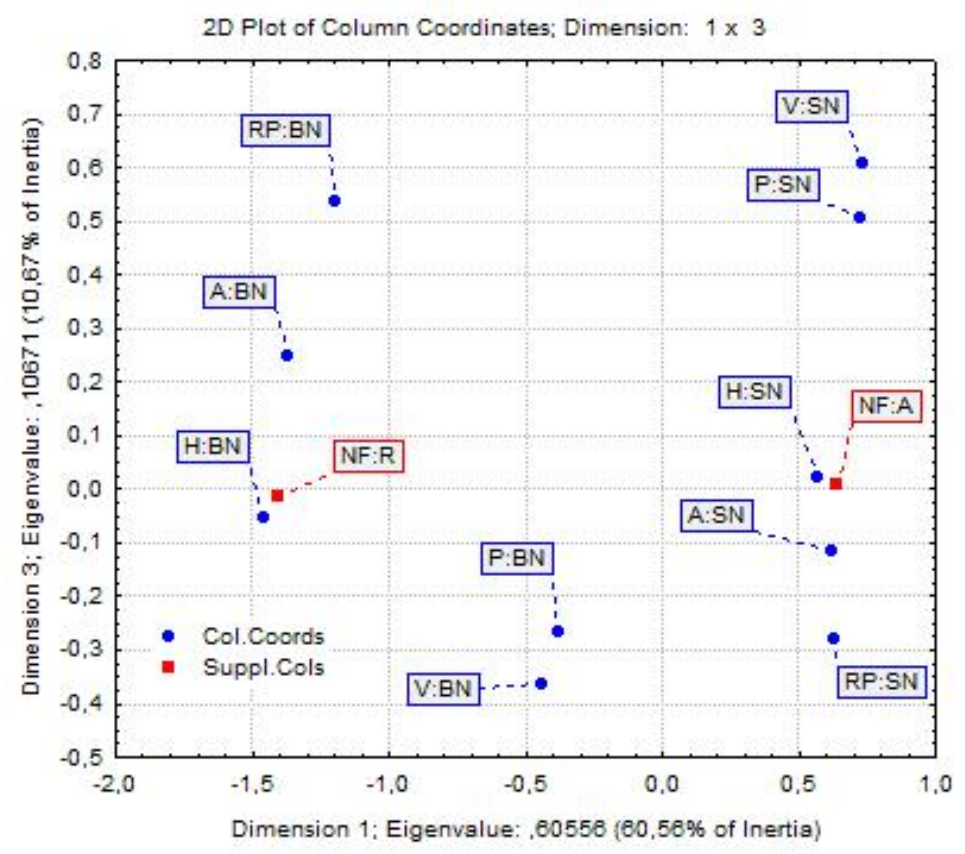

Fig. 6: Muestra la relación entre las variables que afectan el rendimiento académico

Del análisis de la figura 6, se corrobora y evidencia los resultados anteriores en la dimensión 1 , donde nuevamente se contrapone la nota final aprobada NFA, RPSN, HSN; PBN y VSN con la nota final reprobada NFR, RPBN, HBN. PBN y VBN que son las categorías que más aportan a la dimensión 1. Al analizar la dimensión tres se observan dos nubes de puntos en los cuadrantes II y IV, en el cuadrante II se agrupan los alumnos NFR, RPBN y VBN al que se contrapone en cuadrante IV NFA, RPSN, PSN y VSN, también aportan en menor medida en este eje las categorías ASN y HSN ubicada en Cuadrante I, al que se contraponen ABN y HBN que se ubican en cuadrante III, en esta dimensión las variables que más aporta es ASN al que se contrapone ABN.

Se ha aplicado un ACM a la tabla de contingencia donde los dos primeros factores explican aproximadamente un $81 \%$ de la inercia total. Considerando el tercer factor, se explica un 92\%, suficiente para considerar estas tres dimensiones que se corresponden en gran medida con la diferenciación entre los estudiantes que aprueban o repruebas (rendimiento académico) y el desarrollo de las diversas categorías del pensamiento crítico.

\section{CONCLUSIONES}

La relación entre renovación metodológica y el desarrollo de pensamiento crítico es un problema de investigación incipiente. En este sentido este estudio empírico es un avance para investigaciones futuras que traten de acumular evidencias en esta línea de trabajo. Aun cuando los resultados informan de evidencias suficientes como para afirmar la existencia de una relación positiva entre la intervención en una asignatura de Física con ASARPIC y el desarrollo del pensamiento crítico en un semestre académico, no es posible establecer juicios categóricos dado a la gran cantidad de variables intervinientes. Ciertamente el desarrollo de habilidades cognitivas vinculadas al pensamiento crítico exige tener presente el tiempo de intervención que contribuye en 
gran medida a que se produzca esta relación positiva y un enfoque multidisciplinario con renovación metodológica en un mayor número de asignaturas simultáneamente, en este contexto se infiere que cualquier programa destinado a desarrollar el pensamiento crítico en la educación superior, debe considerar renovación metodológica y el tiempo de intervención.

Con relación a la propuesta metodológica de aula y su influencia en el rendimiento académico se plantea la siguiente aseveración de conocimiento acorde con los resultados obtenidos del análisis estadístico de la t de Student, antes de la intervención metodológica en el GC y GE, se verifica que no existen diferencias estadísticamente significativas entre los grupos. Después de la intervención metodológica en ambos grupos GE y GC se registran diferencias estadísticamente significativas en el rendimiento académico (calificación) obtenido en el transcurso del semestre es decir, los resultados alcanzados no son similares en los dos grupos, lo que se corrobora por medio del análisis estadístico, de la prueba t de Student que establece un estadístico $\mathrm{t}=9.23 \mathrm{con}$ un nivel de significado de $p=0,000,99,9 . \%$, las diferencias en el rendimiento académico final son estadísticamente significativa a favor del grupo experimental. De aquí se establece el ASARPIC impacta positivamente en el rendimiento académico de los estudiantes del GE.

A partir del ACM se puede inferir que la propuesta de renovación ASARPIC impacta positivamente las habilidades de pensamiento crítico, especialmente las vinculadas a la resolución de problemas; que implica comprender, transferir, interpretar y clasificar información necesaria para alcanzar un aprendizaje significativo con sentido basado en la relación entre el conocimiento previo y el nuevo conocimiento; también hay evidencias de una fuerte relación entre las categorías del pensamiento crítico y el rendimiento académico, de donde se deduce que los estudiantes utilizan las habilidades del pensamiento crítico para construir conocimientos como son: la capacidad de plantear preguntas y problemas de manera clara y precisa (objetivos de aprendizaje, identificación contenidos previos y a investigar), recopilar y evaluar información relevante y compleja (contenidos a investigados), alcanzar la solución de los problemas (razonar la respuesta), analizar en profundidad sus respuestas y contenidos a abordar (comprobar), y comunicar de forma efectiva sus resultados.

El ASARPIC promueve el trabajo en equipos colaborativos, se facilitan los procesos de reconciliación integradora y la diferenciación progresiva, ya que se evita la fragmentación de los contenidos al enfrentase en primer lugar al todo y luego de forma sucesiva a las partes, logrando la reconciliación de los contenidos, en otras palabras, los problemas y sus contenidos se van diferenciando progresivamente, de los conceptos más generales a los más simples, hasta alcanzar la complejidad y abstracción a través del proceso de diferenciación. Aquí los alumnos tienen ocasión de desarrollar sus destrezas de comunicación y de trabajo en grupo, donde deben aprender a escuchar, explicar, preguntar, responder y, en algunas clases, comunicar sus resultados. Uno de los beneficios reales de esta propuesta es el trabajo en pequeños Grupos donde se anima encarecidamente a los estudiantes a que aprendan unos de otros a través de puestas en común de sus puntos de vista, lo que los ayuda a comprender las teorías o explicaciones complejas, al compartir significado entre el material educativo, alumnos y docente. Los resultados obtenidos muestran que no tiene sentido enseñar sin tener en cuenta el conocimiento previo de los alumnos en alguna medida y que son las situaciones las que dan sentido a los nuevos conocimientos; son los problemas los que permite crear más espacios para que los alumnos expliciten los significados aprendidos, y evidencien su aprendizaje a través de la transferencia de contenidos a situaciones nuevas.

\section{AGRADECIMIENTOS}

La presente investigación es posible gracias al financiamiento logrado a través del proyecto de investigación FONDECYT 1090618.

\section{REFERENCIAS}

Ausubel, D.P, J. D. Novak y H. Hanesian. Psicología Educativa: un punto de vista cognitivo. Trillas. México. (1997). 
Ausubel, D. P. Adquisición y retención del conocimiento. Una perspectiva cognitiva. Paidós. Barcelona. España. (2000).

Becerra, C., A. Gras-Marti y J. Martinez. ¿De verdad se enseña a resolver problemas en el primer curso de física universitaria? la resolución de problemas de "lápiz y papel" en cuestión, Rev. Bras. Enseñanza de la Física 27(1), 299-308 (2005).

Cohen, L. y L. Manion. Métodos de investigación educativa. La muralla S.A. Madrid. España. 1990 Driver, R., A. Squires, P. Rushworth y V. Wood. Making sense of secondary science. Research into children's ideas. New York: Routledge. (1994).

Duch, B. J., S. E. Grogh y D. E. Allen. El poder del aprendizaje basado en problemas. Una guía práctica para la enseñanza universitaria. Editorial Pont. Universidad Católica. Lima. Perú. (2004). López, E., E. Barajas y R. Pérez. "Pedagogía Experimental I". Tomo II, pp. 297-412. UNED. Madrid, España. (1997).

Garret, R. M. Resolución de problemas y creatividad: Implicaciones para el currículum de ciencias. Enseñanza de las ciencias 6(3), 224-230 (1988).

Halpern, D.F. Teaching critical thinking for transfer across domains. American Psychologist, 53, 449-455 (1998).

Halpern, D.F. Thought and knowledge: An introduction to critical thinking (4th ed). Hillsdale, NJ: Erlbaum. (2003).

Halpern, D. Critical Thinking Assessment Using Everyday Situations: Background and scoring standards ( $2^{\circ}$ Report). Unpublished manuscript. College. (2006).

Herrera, E. D. y I. R. Sánchez, Unidad didáctica para abordar el concepto de célula desde la resolución de problema por investigación.. PARADIGMA, 33(1), 63-85 (2009).

Morales, E. P. Logros en la implementación de modalidades híbridas de ABP. Revista de Docencia Universitaria, 9 (1), 67-90 (2011).

Moreira, M.A Por qué conceptos? ¿Por qué aprendizaje significativo? ¿Por qué actividades colaborativas? y ¿Por qué mapas conceptuales? Curriculum, 23(1), 9-23. (2010).

Moreira, M.A Aprendizaje Significativo Crítico, Instituto de Física Universidad Federal de Porto Alegre, Brasil. (2005).

Moreira, M.A. teoria da aprendizagem significativa e sua implementação em sala de aula, (Editora da UnB, Brasília, (2006).

Nieto, A. M., C. Saiz y B. Orgaz, Análisis de las propiedades psicométricas de la versión española del HCTAES-Test de Halpern para la evaluación del pensamiento crítico mediante situaciones cotidianas. Revista Electrónica de Metodología Aplicada, 14 (1), 1-15. (2009).

Novak, J. D. y D. B. Gowin, Aprendiendo a aprender.: Ediciones Martínez Roca, S.A. Barcelona, España. 117-134 (1988).

Oñate, R. J. e I. R. Sánchez. Resolución de problemas por investigación y su influencia en los trabajos prácticos de laboratorio en termodinámica. Revista Pedagogía. 89(2). 307-329 (2010).

Perales, F. J. y A. Salinas. Percepción de estudiantes de física sobre la dificultad en la resolución de problemas, Acta del XXI, Encuentro de didáctica de las ciencias experimentales, España. (2004). 
Pozo, J. I., M. Pérez, J., Domínguez, M. A. Gómez, y Y. Postigo,. Solución de problemas: Santillana/ Aula XXI. Madrid. España. (1994).

Ribeiro, L.R. y M. Mizukami. Problem-based learning, a student evaluation of an implementation in postgraduate engineering education. European Journal of Engineering Education 30(1),137-149 (2005).

Sáenz. O. et al. "Didáctica General”: Un enfoque curricular: Marfil. Alcoy: España. (1998).

Said. S. M., F.R. Mahamd., S. Mekhilef y N. A. Rahim. Implementation of the problem-based learning approach in the Department of Electrical Engineering, University of Malaya. European Journal of Engineering Education 30(1), 129-136 (2005).

Sánchez, I.R, L. Neriz y F.J. Ramis. Design and application of learning environments based on integrative problems. European Journal of Engineering Education. 33(4), 445-452 (2008).

Sánchez I.R., M.A. Moreira, y M.C. Caballero. "Implementación de una renovación metodológica para un aprendizaje significativo en Física I". Latin American Journal of Physics Education. 5(2), 475-484 (2011).

Sánchez, I. R., M.A. Moreira y M. C. Caballero, Implementación de una propuesta de aprendizaje significativo de la cinemática a través de la resolución de problemas. Revista chilena de ingeniería, 17(1), 27-41 (2009).

Sánchez, I. R. Propuesta de aprendizaje significativo a través de resolución de problemas por investigación, Revista Educere. 47(3), 947-959 (2009a).

Sánchez, I. R. Influencia de la resolución de problemas por investigación; en el pensamiento crítico, estrategias y calidad del aprendizaje, Enseñanza de las Ciencias. Número Extra, VIII Congreso Internacional sobre Investigación en Didáctica de las Ciencias, Barcelona, 3503-3507. (2009b).

Sánchez, I. R. y P. A. Flores. Influencia de una metodología activa en el proceso de enseñar y aprender Física. Journal of Sciencie Education, 5 (2), 77-83 (2004).

Sierra, R. Técnicas de Investigación Social. Ed. Paraninfo. Madrid, España. (2002).

Vigotsky, L. El desarrollo de los procesos psicológicos superiores. Grijalbo. Barcelona. España (1979). 